\title{
Systematic Effects at Criticality for the SU(2)-Landau-Gauge Gluon Propagator
}

\section{Tereza Mendes*}

Instituto de Física de São Carlos, Universidade de São Paulo, Caixa Postal 369, 13560-970 São Carlos, SP, Brazil

E-mail: mendesdifsc.usp.br

\section{Attilio Cucchieri}

Instituto de Física de São Carlos, Universidade de São Paulo, Caixa Postal 369, 13560-970 São Carlos, SP, Brazil

E-mail: attiliodifsc.usp.br

We analyze data from finite-temperature simulations of the gluon propagator in SU(2) Landau gauge on large lattices. We argue that the singular behavior of this quantity around the deconfinement transition, seen in several previous studies, is a lattice artifact.

31st International Symposium on Lattice Field Theory - LATTICE 2013

July 29 - August 3, 2013

Mainz, Germany

\footnotetext{
*Speaker.
} 


\section{Introduction}

The gluon propagator is the most fundamental quantity of QCD and its infrared behavior is believed to be closely related to the phenomenon of color confinement in the theory. In particular, the Gribov-Zwanziger confinement scenario in Landau gauge [1] predicts a suppressed gluon propagator in the infrared limit (in combination with an enhanced ghost propagator). According to this scenario, in fact, the gluon propagator should go to zero in the limit of vanishing momentum. These predictions are investigated by approximate analytic methods such as Dyson-Schwinger equations and functional renormalization group calculations. At the same time, the lattice formulation can provide valuable insight into the problem and numerical checks of the predictions. Unfortunately, the infrared limit corresponds to large lattice sizes, which are computationally demanding. This issue has turned out to be particularly challenging in Landau gauge, requiring numerical investigations using the largest lattices ever considered (see [2] for a review). Nevertheless, the infrared limit may be qualitatively studied for pure SU(2) gauge theory and, at the same time, using very large lattices might greatly reduce the infamous problem of gauge-fixing ambiguity due to Gribov copies [3].

Lattice simulations have established that the momentum-space gluon propagator $D\left(p^{2}\right)$ is suppressed in the limit of small momentum $p$, while the real-space gluon propagator violates reflection positivity. This latter feature, consistent with gluon confinement, is observed for all lattice volumes. On the other hand, whereas a fit of $D\left(p^{2}\right)$ to the Gribov form is possible at moderate lattice volumes, data obtained using very large lattices (of linear size $L \approx 27 \mathrm{fm}$ ) revealed that $D(0)$ is strictly nonzero. This behavior has been termed "massive", since it may be interpreted as a dynamically generated mass for the gluon, and was first proposed as a solution to the Dyson-Schwinger equations of QCD in [4]. Several variants of such massive behavior have been used to fit lattice data for the Landau-gauge gluon propagator. In particular, in [5], very good fits to rational (or Gribov-Stingl) forms were obtained in the four-dimensional case, as well as for three space-time dimensions. These fits are shown in Fig. 1. The fitting forms in the 4D and in the 3D cases are given respectively by

$$
D_{1}\left(p^{2}\right)=C \frac{p^{2}+d}{p^{4}+u^{2} p^{2}+t^{2}}
$$

and

$$
D_{2}\left(p^{2}\right)=C \frac{\left(p^{2}+d\right)\left(p^{2}+1\right)}{\left(p^{4}+u^{2} p^{2}+t^{2}\right)\left(p^{2}+v\right)},
$$

corresponding (respectively) to three and to four free parameters, in addition to the global normalization constant $C$. Noting that the three-dimensional case may be viewed as the infinitetemperature limit of the four-dimensional case in the transverse sector, one may be motivated to look for an interpolation of the above 4D and 3D zero-temperature forms to describe the finitetemperature data for the propagator.

In this contribution we present final results of our numerical study of the finite-temperature gluon propagator in the electric sector. We focus on the infrared value of the longitudinal propagator $D_{L}\left(p^{2}\right)$ as a function of the temperature. A detailed analysis of our data will be presented elsewhere [6]. (Preliminary results were reported in $[7,8,9,10]$. 

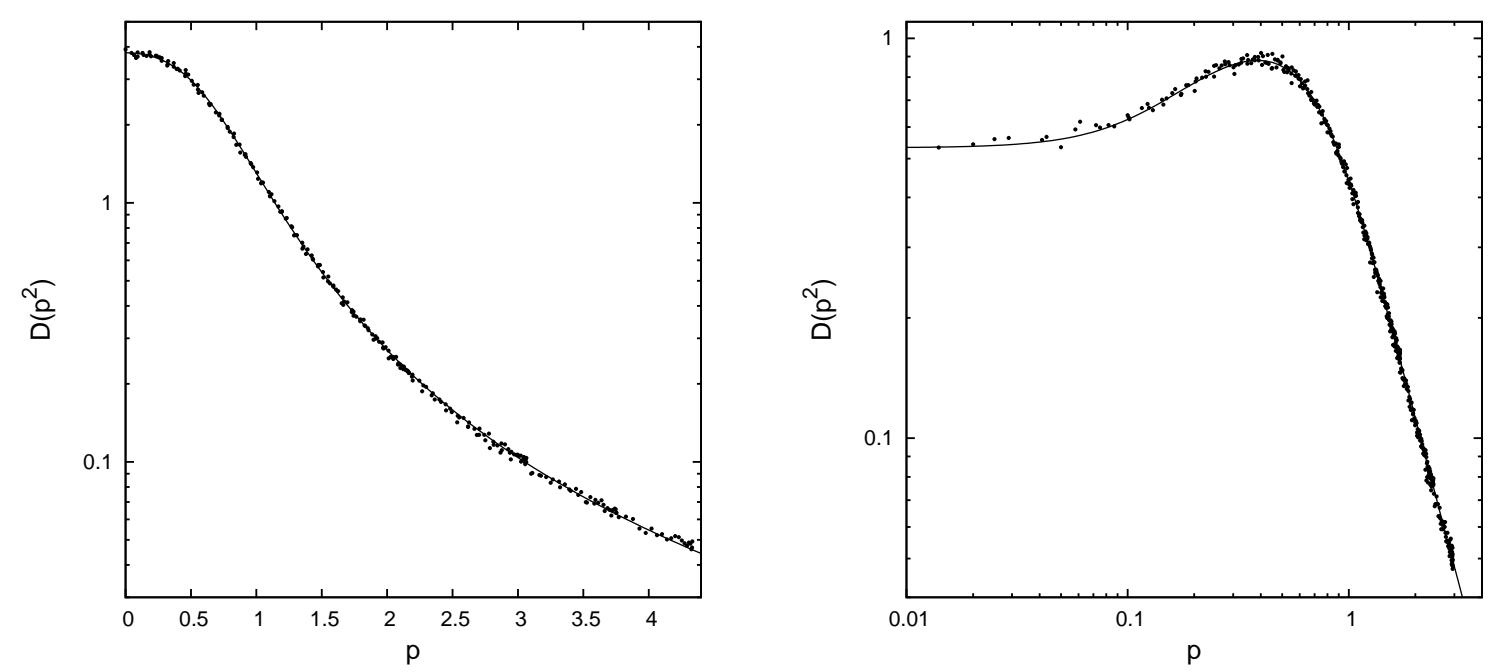

Figure 1: Fits of zero-temperature data for the SU(2) Landau-gauge gluon propagator to rational (GribovStingl) forms in the 4D (left) and 3D (right) cases. Plots extracted from [5].

\section{Gluon propagator at finite temperature}

As the temperature $T$ is turned on, we expect to observe Debye screening of the color charge. In particular, at high temperatures, deconfinement should be felt in the longitudinal (i.e. electric) gluon propagator as an exponential fall-off at long distances, defining a screening length and conversely a screening mass [11]. It is not clear how such a mass would show up around the critical temperature $T_{c}$. At the same time, as discussed above, studies of the gluon propagator at zero-temperature have shown a (dynamical) mass. One can try to use this knowledge to define temperature-dependent masses for the region around $T_{c}$. Conversely, the dimensional-reduction picture (based on the 3D-adjoint-Higgs model) suggests a confined magnetic gluon, associated to a nontrivial magnetic mass. This mass should in turn be obtained from the infrared behavior of the transverse gluon propagator.

Lattice studies of the Landau-gauge gluon propagator around the deconfinement phase transition in pure $S U(2)$ and $S U(3)$ theory, as well as considering dynamical quarks, have been presented in $[12,13,14,15,16,17,18,19]$. In the transverse (i.e. magnetic) sector, one sees strong infrared suppression of the propagator, with a turning point of the curve described by the momentum-space magnetic propagator $D_{T}\left(p^{2}\right)$ for momenta $p$ around $400 \mathrm{MeV}$. This suppression seems even more pronounced than in the zero-temperature case discussed in the Introduction. Also, $D_{T}\left(p^{2}\right)$ shows considerable finite-physical-size effects in the infrared limit, as observed for $T=0$. Furthermore, just as for $T=0$, the magnetic propagator displays a clear violation of reflection positivity in real space. Essentially these same features are seen for $D_{T}\left(p^{2}\right)$ at all nonzero temperatures considered.

The longitudinal propagator $D_{L}\left(p^{2}\right)$, on the other hand, shows significantly different behavior for different temperatures. As soon as a nonzero temperature is introduced in the system, $D_{L}\left(p^{2}\right)$ increases considerably (whereas $D_{T}\left(p^{2}\right)$ decreases monotonically). More precisely, for all fixed temperatures, the curve described by $D_{L}\left(p^{2}\right)$ seems to reach a plateau in the low-momentum region (see e.g. [8]). As the temperature is increased, this plateau increases slightly until, approaching 
the phase transition from below, it has been observed to rise further and then, just above the transition temperature, to drop sharply. This has been interpreted as a sign of singular behavior of the longitudinal gluon propagator around $T_{c}$ and, in fact, it has been related to several proposals of a new order parameter for the deconfinement transition. (Of course, a relevant question is, then, whether this singularity survives the inclusion of dynamical quarks in the theory [17, 18].)

Let us mention that, at all investigated temperatures, the infrared plateau just described is not long enough to justify a fit to the Yukawa form

$$
D_{L}\left(p^{2}\right)=C \frac{1}{p^{2}+m^{2}},
$$

predicted at high temperatures. If this were the case, $D_{L}(0)^{-1 / 2}$ would provide a natural (temperature-dependent) mass scale. Note that this value depends also on the global constant $C$. On the other hand, Gribov-Stingl forms such as in Eqs. (1.1) and (1.2) above involve complex-conjugate poles, defining real and imaginary masses (independently of $C$ ). Here we do not show data (or fits) for $D_{L}\left(p^{2}\right)$. Such curves and (preliminary) fits can be seen e.g. in [9]. Instead, we will look at the value of $D_{L}(0)$ (after normalization by $C$ ) as a function of $T$.

Concerning the longitudinal propagator in real space (see e.g. [9]), positivity violation is observed unequivocally only at zero temperature and for a few cases around the critical region, in association with the severe systematic errors discussed below. For all other cases, there is no violation within errors. Also, we always observe an oscillatory behavior, indicative of a complex-mass pole. In the next section, we present our new results for the infrared values of $D_{L}\left(p^{2}\right)$.

\section{Results}

Our large-lattice study was done considering the pure $\mathrm{SU}(2)$ case, with a standard Wilson action and lattice sizes $N_{s}^{3} \times N_{t}$ ranging from $48^{3} \times 4$ to $192^{3} \times 16$. For our runs we employ a cold start, performing a projection on positive-Polyakov-loop configurations. Also, gauge fixing is implemented using stochastic overrelaxation. The gluon dressing functions are normalized to 1 at $2 \mathrm{GeV}$. We considered several values of the lattice parameter $\beta$, allowing a broad range of temperatures. Our procedure for determining the physical temperature $T$ is described in [8]. The momentum-space expressions for the transverse and longitudinal gluon propagators $D_{T}\left(p^{2}\right)$ and $D_{L}\left(p^{2}\right)$ can be found e.g. in [12].

As can be seen from the data in [9], the longitudinal (electric) propagator $D_{L}\left(p^{2}\right)$ displays severe systematic effects around $T_{c}$ for the smaller values of $N_{t}$. These effects are strongest at temporal extent $N_{t}=4$ and large values of $N_{s}$. We note that the systematic errors for small $N_{t}$ come from two different sources: "pure" small- $N_{t}$ effects (associated with discretization errors) and strong dependence on the spatial lattice size $N_{s}$ at fixed $N_{t}$, for the cases in which the value of $N_{t}$ is smaller than 16 . The latter effect was observed only at $T \lesssim T_{c}$, whereas the former is present in a wider range of temperatures around $T_{c}$. In particular, the finite-spatial-volume effects for $D_{L}\left(p^{2}\right)$ at $N_{t}=4$ are strongest at $T_{c}$, but are still very large at $T=0.98 T_{c}$ and are much less pronounced for $T=1.01 T_{c}$.

In Fig. 2 we show data for $D_{L}(0)$ as a function of the temperature $T$. We show such values as obtained from all our runs, grouping together (by color) the runs performed at the same temporal 


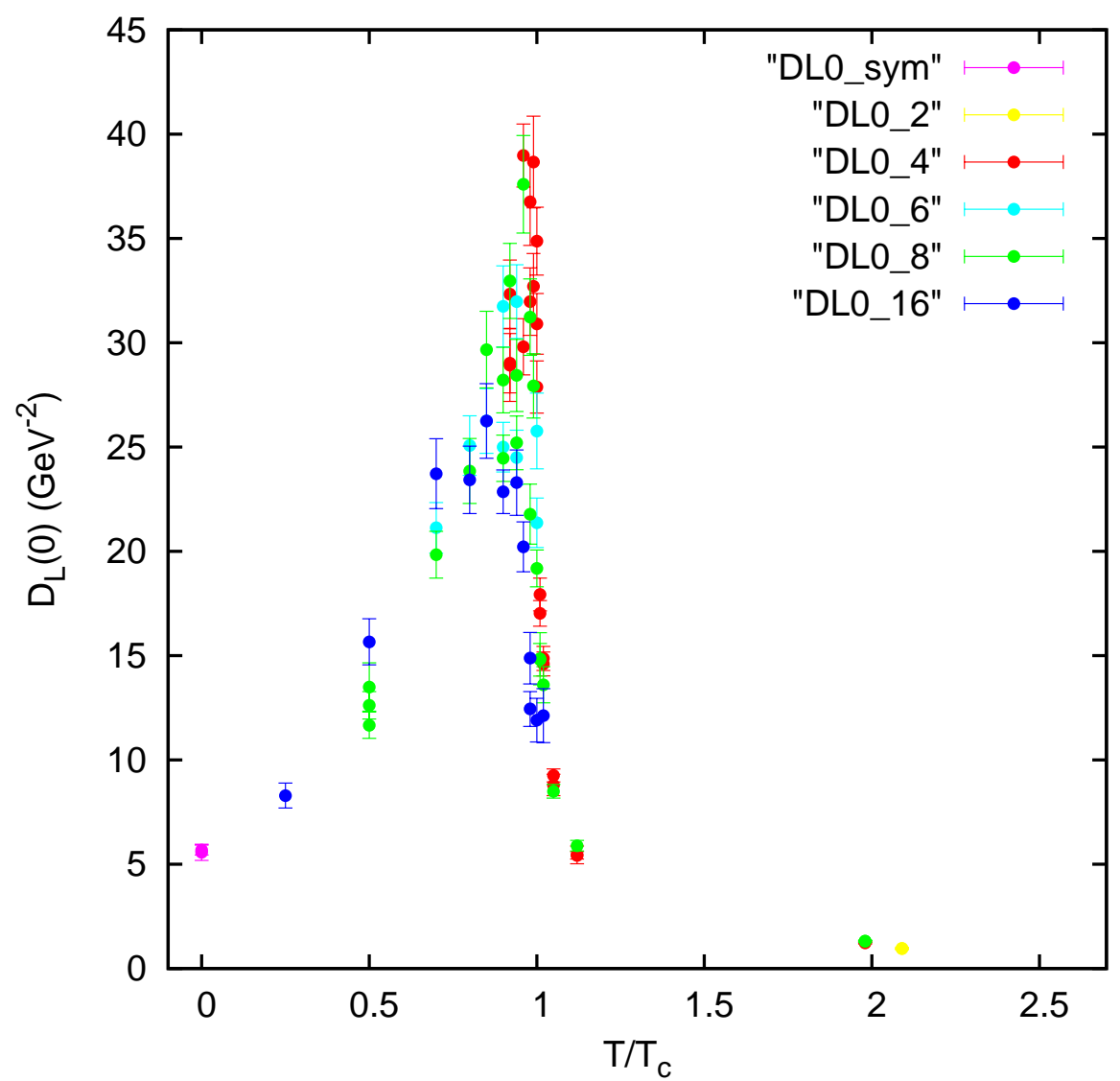

Figure 2: Infrared-plateau value for the longitudinal gluon propagator [estimated by $D_{L}(0)$ ] as a function of the temperature for the full range of $T / T_{c}$ values. Data points from runs at the same value of $N_{t}$ are grouped together and indicated by the label "DL0_N $N_{t}$ ", where "sym" is used to indicate symmetric lattices (i.e. $T=0$ ).

extent $N_{t}$. We remark that, as said above, not all curves of $D_{L}\left(p^{2}\right)$ reach a clear plateau in the infrared limit. Nevertheless, looking at the value of $D_{L}(0)$ gives us an indication of what this plateau might be, and is useful to expose the strong systematic effects discussed here. In Fig. 3 we present an enlarged view of the same values for the temperature region around $T_{c}$.

We can see that the very suggestive sharp peak at $T_{c}$ seen for $N_{t}=4$ (corresponding to the red points in Figs. 2 and 3) turns into a finite maximum around $0.9 T_{c}$ for $N_{t}=16$ (blue points). In other words, the observed singularity at smaller values of $N_{t}$ seems to disappear. The only indication of a possible singular behavior is a finite maximum close to (but not at) the critical point, somewhat reminiscent of a pseudo-critical point as observed for the magnetic susceptibility of spin models in an external magnetic field (see e.g. [20, 21]).

Let us mention that, as reported in [9], good fits are obtained (in the transverse and longitudinal cases) to several generalized Gribov-Stingl forms, indicating the presence of comparable real and imaginary parts of pole masses. These masses are smooth functions of $T$ around the transition, and the imaginary part of the electric mass seems to get smaller at higher $T$, as expected. 


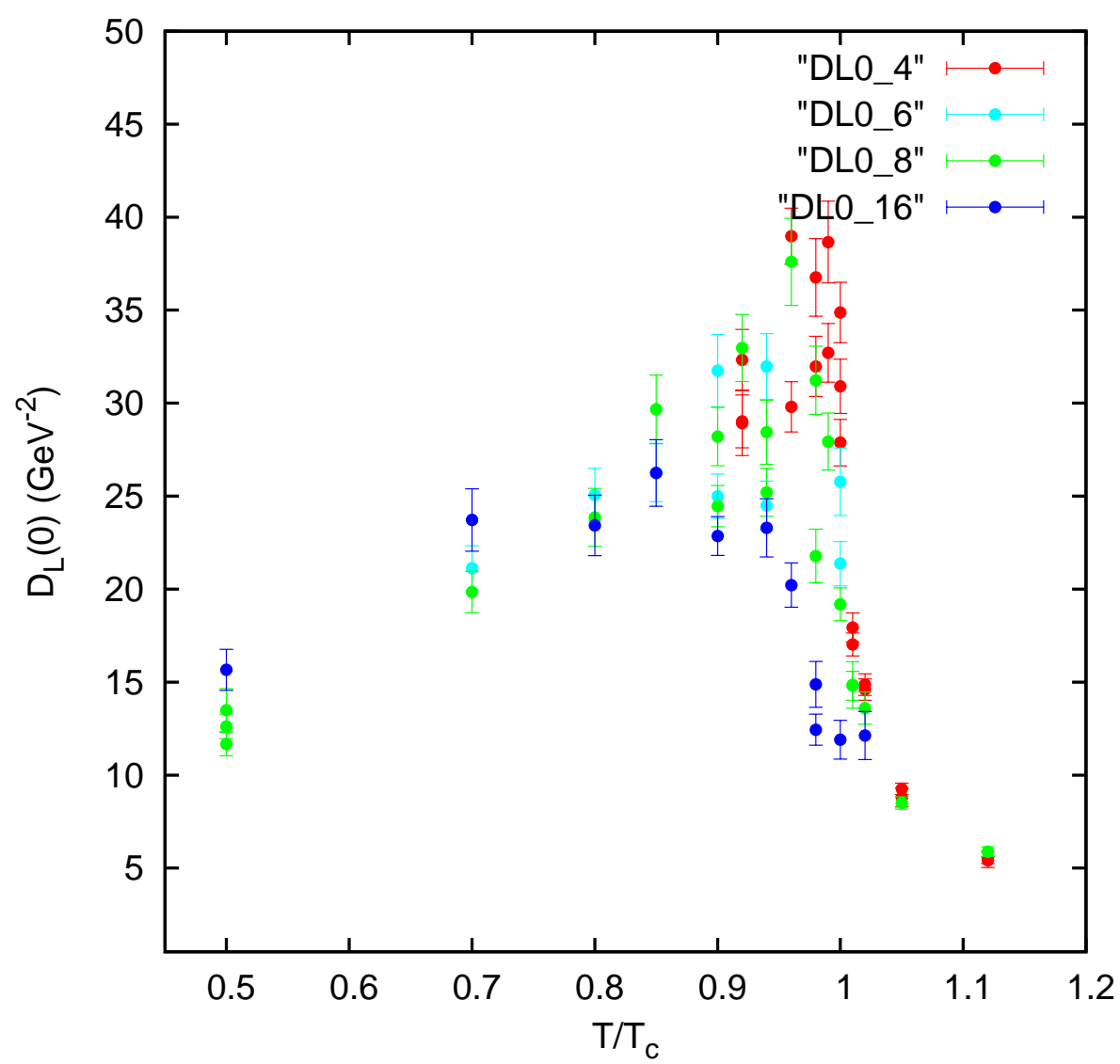

Figure 3: Same as Fig. 2 above, but showing only the temperature region around $T_{c}$.

\section{Conclusions}

We have performed numerical simulations of the longitudinal (electric) and transverse (magnetic) Landau-gauge gluon propagator at nonzero temperature for pure SU(2) lattice gauge theory. We employ the largest lattices to date, especially for temperatures around the deconfinement phase transition. We are currently completing our study of fitting forms for describing the massive behavior of the propagator [6]. From our data for the longitudinal gluon propagator $D_{L}\left(p^{2}\right)$, we have uncovered quite severe systematic effects.

Our results point to unusually large systematic errors around criticality. In particular, very strong effects related to small values of the temporal extent $N_{t}$ of the lattice are seen on the lower side of the transition temperature and are practically absent just above $T_{c}$. Strong finite-size effects are certainly not unexpected around a second-order phase transition, such as the deconfinement transition in the SU(2) theory. On the other hand, we note that our data show a nontrivial dependence on the finite temporal size of the lattice and on the distance from the critical point, not easily interpreted as a finite-size or a discretization effect.

After removing these systematic effects, i.e. considering the data obtained with the largest value of $N_{t}$ in Fig. 3, we see that the sharp peak suggested by the red points in Fig. 2 turns into 
a smooth maximum, at around $0.9 T_{c}$. In agreement with several observations that the gluon mass scale is a smooth function of the temperature, this suggests that there is no specific signature of deconfinement associated with $D_{L}\left(p^{2}\right)$. In fact, the only qualitative feature of a deconfined phase we observe is the lack of violation of reflection positivity for the real-space electric propagator, which holds however for all $T \neq 0$ considered.

Finally, let us mention the similarity between our smaller-lattice results for the SU(2) case and existing results for $\mathrm{SU}(3)$. In view of this, we trust that statements that the inverse of the zeromomentum value of the gluon propagator might provide an order parameter for the deconfinement phase transition (such as recently made in [19]) will be taken with the due caution.

\section{References}

[1] N. Vandersickel and D. Zwanziger, Phys. Rept. 520, 175 (2012) [arXiv:1202.1491 [hep-th]].

[2] A. Cucchieri and T. Mendes, PoS QCD -TNT09, 026 (2009) [arXiv:1001.2584 [hep-lat]].

[3] D. Zwanziger, Phys. Rev. D 69, 016002 (2004) [hep-ph/0303028].

[4] J. M. Cornwall, Phys. Rev. D 26, 1453 (1982).

[5] A. Cucchieri, D. Dudal, T. Mendes and N. Vandersickel, Phys. Rev. D 85, 094513 (2012) [arXiv:1111.2327 [hep-lat]].

[6] A. Cucchieri and T. Mendes, in preparation.

[7] A. Cucchieri and T. Mendes, PoS LATTICE 2010, 280 (2010) [arXiv:1101.4537 [hep-lat]].

[8] A. Cucchieri and T. Mendes, PoS FACESQCD, 007 (2010) [arXiv:1105.0176 [hep-lat]].

[9] A. Cucchieri and T. Mendes, PoS LATTICE 2011, 206 (2011) [arXiv:1201.6086 [hep-lat]].

[10] A. Cucchieri, D. Dudal, T. Mendes and N. Vandersickel, PoS QCD-TNT-II, 030 [arXiv:1202.0639 [hep-lat]].

[11] D. J. Gross, R. D. Pisarski, L. G. Yaffe, Rev. Mod. Phys. 53, 43 (1981).

[12] A. Cucchieri, A. Maas, T. Mendes, Phys. Rev. D 75, 076003 (2007) [arXiv:hep-lat/0702022].

[13] C. S. Fischer, A. Maas, J. A. Muller, Eur. Phys. J. C 68, 165 (2010) [arXiv:1003.1960 [hep-ph]].

[14] V. G. Bornyakov and V. K. Mitrjushkin, Phys. Rev. D 84, 094503 (2011) [arXiv:1011.4790 [hep-lat]].

[15] R. Aouane, V. G. Bornyakov, E. M. Ilgenfritz, V. K. Mitrjushkin, M. Muller-Preussker and A. Sternbeck, Phys. Rev. D 85, 034501 (2012) [arXiv:1108.1735 [hep-lat]].

[16] A. Maas, J. M. Pawlowski, L. von Smekal and D. Spielmann, Phys. Rev. D 85, 034037 (2012) [arXiv:1110.6340 [hep-lat]].

[17] V. G. Bornyakov and V. K. Mitrjushkin, Int. J. Mod. Phys. A 27, 1250050 (2012) [arXiv:1103.0442 [hep-lat]].

[18] R. Aouane, F. Burger, E. -M. Ilgenfritz, M. Muller-Preussker and A. Sternbeck, Phys. Rev. D 87, 114502 (2013) [arXiv:1212.1102 [hep-lat]].

[19] P. J. Silva, O. Oliveira, P. Bicudo and N. Cardoso, arXiv:1310.5629 [hep-lat].

[20] A. Cucchieri and T. Mendes, J. Phys. A 38, 4561 (2005) [hep-lat/0406005].

[21] J. Engels and F. Karsch, Phys. Rev. D 85, 094506 (2012) [arXiv:1105.0584 [hep-lat]]. 